\title{
Identification of a nuclear localization motif in the serine/arginine protein kinase PSRPK of physarum polycephalum Shide Liu ${ }^{\dagger 1}$, Zhuolong Zhou ${ }^{\dagger 1}$, Ziyang Lin ${ }^{2}$, Qiuling Ouyang1, Jianhua Zhang1, Shengli Tian ${ }^{1}$ and Miao Xing*1
}

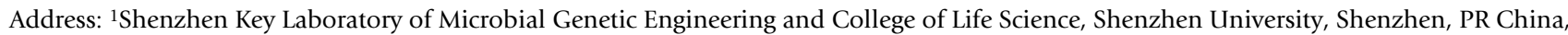
518060 and Institute of Photoelectron, Shenzhen University, Shenzhen, PR China, 518060

Email: Shide Liu - liusd@szu.edu.cn; Zhuolong Zhou - zhouzhuolong@163.com; Ziyang Lin - lzy@szu.edu.cn; Qiuling Ouyang - isabel_ou@163.com; Jianhua Zhang - zhangjh8@szu.edu.cn; Shengli Tian - sltian@szu.edu.cn; Miao Xing* - xingmiao@szu.edu.cn

* Corresponding author †Equal contributors

Published: 25 August 2009

BMC Biochemistry 2009, 10:22 doi:10.1/86/147|-2091-10-22
Received: 28 February 2009

Accepted: 25 August 2009

This article is available from: http://www.biomedcentral.com/I47I-209|/I0/22

(c) 2009 Liu et al; licensee BioMed Central Ltd.

This is an Open Access article distributed under the terms of the Creative Commons Attribution License (http://creativecommons.org/licenses/by/2.0), which permits unrestricted use, distribution, and reproduction in any medium, provided the original work is properly cited.

\begin{abstract}
Background: Serine/arginine (SR) protein-specific kinases (SRPKs) are conserved in a wide range of organisms, from humans to yeast. Studies showed that SRPKs can regulate the nuclear import of SR proteins in cytoplasm, and regulate the sub-localization of SR proteins in the nucleus. But no nuclear localization signal (NLS) of SRPKs was found. We isolated an SRPK-like protein PSRPK (GenBank accession No. DQ 140379) from Physarum polycephalum previously, and identified a NLS of PSRPK in this study.

Results: We carried out a thorough molecular dissection of the different domains of the PSRPK protein involved in its nuclear localization. By truncation of PSRPK protein, deletion of and single amino acid substitution in a putative NLS and transfection of mammalian cells, we observed the distribution of PSRPK fluorescent fusion protein in mammalian cells using confocal microscopy and found that the protein was mainly accumulated in the nucleus; this indicated that the motif contained a nuclear localization signal (NLS). Further investigation with truncated PSPRK peptides showed that the NLS (318PKKGDKYDKTD ${ }^{328}$ ) was localized in the alkaline $\Omega$-loop of a helix-loophelix motif (HLHM) of the C-terminal conserved domain. If the ${ }^{318} \mathrm{PKK} \mathrm{KDK}^{322}$ sequence was deleted from the loop or $\mathrm{K}^{320}$ was mutated to $\mathrm{T}^{320}$, the PSRPK fluorescent fusion protein could not enter and accumulate in the nucleus.

Conclusion: This study demonstrated that the ${ }^{318}$ PKKKGDKYDKTD ${ }^{328}$ peptides localized in the C-terminal conserved domain of PSRPK with the $\Omega$-loop structure could play a crucial role in the NLS function of PSRPK.
\end{abstract}

\section{Background}

Serine/arginine (SR) protein-specific kinases (SRPKs) represent a class of evolutionarily conserved kinases that specifically phosphorylate the arginine/serine-rich (RS) domains of the SR splicing factor [1]. After the identifica- tion of SRPK1-the first SRPK-by Gui et al. [2,3], other SRPKs such as SRPK2 [4], mouse SRPK1 and SRPK2 [5], yeast Dsk1 [6] and Sky1p [7], nematode SPK-1 [8], Trypanosoma cruzi TcSRPK [9], and Arabidopsis thaliana SRPK4 [10] have been subsequently identified. Some 
studies have shown that SRPKs are mainly localized in the cytoplasm, with only a few present in the nucleus $[[2,5]$, and [11]]. The SRPKs that are localized in the cytoplasm regulate the nuclear import of SR proteins via phosphorylation [12], while those in the nucleus regulate the nuclear localization of the SR splicing proteins via phosphorylation [13-15]. Ding et al. [11] have discovered that the spacer sequences present between the conserved domains of mammalian SRPKs have cytoplasmic anchoring function. However, there has been no report on the nuclear localization signal (NLS) of SRPKs.

Model organism Physarum polycephalum is mitochondriacontaining primitive eukaryotes. Its life cycle includes a single-celled amoeba, plasmodium (the main life form), and sporulation stages. The nuclei in the same plasmodium proliferate by way of synchronization of mitosis. In our previous study, we identified an SRPK containing 426 amino acids (aa) from $P$. polycephalum; this kinase was termed PSRPK (GenBank accession No. DQ140379). Similar to other SRPKs, PSRPK also has 2 conserved domains and can phosphorylate human SR protein alternate splicing factor/splicing factor 2 (ASF/SF2). However, it differs from other SRPKs in that the divergent motif $(\geq N)$ in its $\mathrm{N}$-terminus is rich in acidic amino acids; the spacer sequence of PSRPK between two conserved domains is shorter than that of other SRPKs. In this study, the distribution of PSRPK fluorescent fusion protein in mammalian cells was observed using laser scanning confocal microscopy. Maximum fluorescence was detected in the nucleus, suggesting the existence of an NLS in PSRPK. When the distribution of truncated PSPRK peptides was observed, a putative NLS was found in the C-terminal of PSRPK based on its homology to the classic NLS. When the sequence was deleted or when lysine 320 in the sequence was substitute by threonine, the cytoplasmic distribution of PSRPK was observed.

\section{Methods \\ Construction of expression plasmids containing PSRPK and truncated PSPRK peptides}

pMD18-psrpk had been constructed previously [16]. PSRPK fragments were obtained by polymerase chain reaction (PCR), using pMD18-psrpk as the template and PSRPK-specific primers (Table 1). The resulting PCR fragments and the mammalian expression vectors pDsRed 1 $\mathrm{N} 1$ and pECFP-C1 (Clontech) were digested with EcoRI and BamHI. The digested fragments were subjected to agarose gel electrophoresis, recovered from the gel, purified, and ligated using T4 DNA ligase; this resulted in the expression plasmids pDsRed-psrpk and pECFP-psrpk.

We designed the following truncated PSRPK peptides (Figure 1): TP1 ( $\geq N$ deletion), TP2 (spacer sequence and CD2 deletions), TP3 ( $\geq \mathrm{N}$ and CD1 deletions), TP4 ( $\geq \mathrm{N}, \mathrm{CD} 1$, and spacer sequence deletions), TP5 (without the ${ }^{318}$ PKKGDKYDK ${ }^{326}$ and ${ }^{329}$ DHLALMIELLG ${ }^{339}$ sequences), TP6 (containing 318PKKGDKYDK326 and ${ }^{329}$ DHLALMIELLG $^{339}$ sequences), TP7 TP9 (all without the 318PKKGDKYDKTD 328 sequence; further, TP7, 329 426 aa; TP8, 197 317 aa combined with 329 426 aa; TP9, 1 317 aa combined with 329 426 aa). Recombinant plasmids were constructed using the primer pairs (Table 1) and the pMD18-psrpk, pDsRed1-N1, and pECFP-C1 vectors, resulting in pECFP-tp1 pECFP-tp4, pDsRed-tp5, pDsRed-tp6, pDsRed-tp7, pDsRed-tp8, and pDsRed-tp9.

\section{Construction of expression plasmids containing mutant and default PSRPK peptides}

In order to understand the effect of the loop motif on nuclear localization, we designed default PSRPK, namely, PSRPK $^{\mathrm{d}}$ (318PKKGDK ${ }^{323}$ deletion) and mutant PSRPK, namely, PSRPK $\left(\mathrm{K}^{320} \rightarrow \mathrm{T}^{320}\right.$ mutation). The DNA fragments containing $p s r p k^{d}$ or $p s r p k^{m}$ up- and downstream

Table I: Primers used for cloning psrpk and tps

\begin{tabular}{|c|c|c|}
\hline & DNA fragmants & sense and anti-sense primers \\
\hline psrpk & $1 \sim 1278$ & $\begin{array}{l}\text { FI:5'-CCGGAATTCTATGGAAAACATATTCAAGGAGAAGG-3' } \\
\text { RI: 5'-ACGCGTCGACAGAAATGGAGGCACATCAGCC-3' }\end{array}$ \\
\hline$t p l$ & $84 \sim 1278$ & 5'-CCGGAATTCTATGGATAGCGAAGATGAGGGAAC-3'/RI \\
\hline tp2 & $1 \sim 588$ & FI/5'-ACGCGTCGACAGCACGTTTTCAGGTTTGAG-3' \\
\hline tp3 & $589 \sim 1278$ & 5'-CCGGAATTCTATGGACCATCTGTTACGACCAG-3'/RI \\
\hline tp4 & $778 \sim 1278$ & 5'-CCGGAATTCTATGAAAATCGCCGATCTAGGCAC-3'/R I \\
\hline tp5 & $589 \sim 951$ & $\begin{array}{l}\text { F2: 5'-CCGGAATTCATGGACCATCTGTTACGACCAGACAC-3' } \\
\text { R2: 5'-ACGCGTCGACTGGCAAAAAAGGAGATCTCCGGTGG-3' }\end{array}$ \\
\hline tp6 & $589 \sim 1029$ & F2/5'-ACGCGTCGACTGGCGCGGCATTCTTCCTAGTAGCTC-3' \\
\hline tp7 & $985 \sim 1278$ & $\begin{array}{l}\text { F3: 5'-ACGCGTCGACGATGGATCACTTGGCTTTGATGATTG-3' } \\
\text { R3: 5'-ATGCGGATCCGCCAGAAATGGAGGCACATCAGCC-3' }\end{array}$ \\
\hline tp8 & $\begin{array}{l}589 \sim 951 \\
985 \sim 1278\end{array}$ & $\begin{array}{l}\text { F2/R2 } \\
\text { F3/R3 }\end{array}$ \\
\hline tp9 & $\begin{array}{l}|\sim 95| \\
985 \sim 1278\end{array}$ & $\begin{array}{l}\text { F4: 5'-CCGGAATTCATGGAAAACATATTCAAGGAGAAG-3'/R2 } \\
\text { F3/R3 }\end{array}$ \\
\hline
\end{tabular}




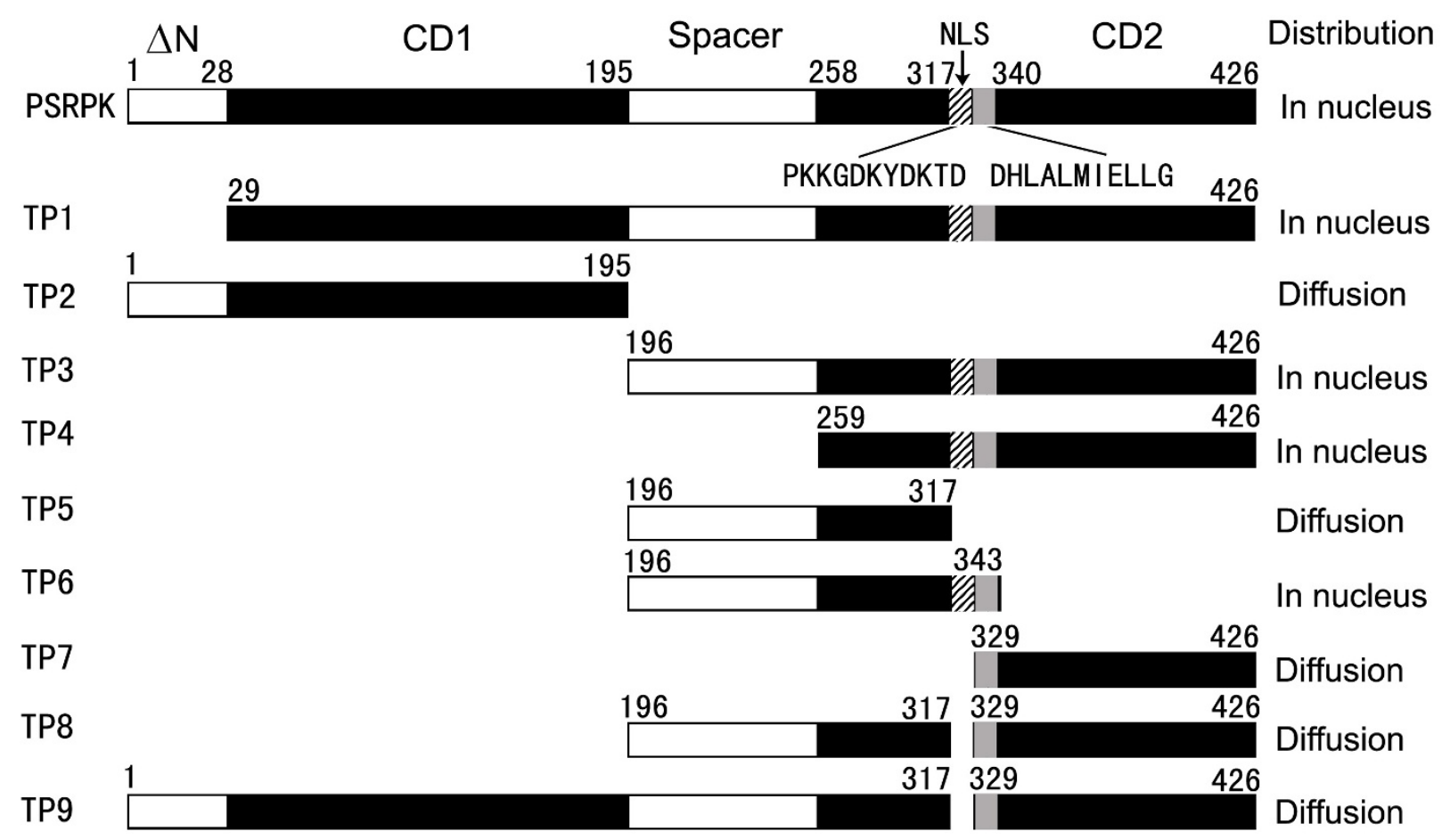

Figure I

Distribution of fluorescent fused PSRPK in mammalian cells. a and b: DAPI staining nuclear of cells HeLa and L929 containing PECFP-psrpk were observed under a BX5I fluorescent microscope (Olympus, 400x); c and d: distribution of CFPPSRPK in cells HeLa and L929 were observed under a confocal microscope (TCS SP2, Leica).

were obtained using the primers listed in Table 2 and pMD18-psrpk as the template. The 2 overlapping PCR products were mixed in a ratio of $1: 1$, followed by denaturing at $94^{\circ} \mathrm{C}$ for $3 \mathrm{~min}$ and annealing at $55^{\circ} \mathrm{C}$ for $3 \mathrm{~min}$. Since there were 18 complementary bases (underlined parts of primers, Table 2) in the 2 PCR fragments, they would be complementary to the cohesive ends during annealing at $55^{\circ} \mathrm{C}$. The DNA with $5^{\prime}$ and $3^{\prime}$ overhangs could be extended by DNA polymerase (Hot Start Taq Polymerase; Qiagen) to form a complete DNA fragment at $72^{\circ} \mathrm{C}$. Primer pair F4/R3 were introduced in the PCR reaction for the amplification of the $p s r p k^{d}$ and $p s r p k^{m}$ sequences. The 2 PCR products, $p s r p k^{d}$ and $p s r p k^{m}$, were inserted into pDsRed1-N1, resulting in the recombinant plasmids pDsRed-psrpk $k^{d}$ and pDsRed-psrpk ${ }^{m}$, respectively.

\section{Transfection of mammalian cells with lipofectamine}

The abovementioned recombinant plasmids were used to transform E. coli DH5a cells. The positive recombinant products were grown in Luria-Bertani (LB) medium containing $30 \mu \mathrm{g} / \mathrm{ml}$ kanamycin. The plasmids were isolated using the alkaline lysis method, dissolved in Tris-EDTA (TE) buffer, and quantified using GeneQuant pro (Amersham Bioscience, GE Healthcare). The purified plasmid $\left(\mathrm{A}_{260} / \mathrm{A}_{280}>1.8\right)$ was diluted to $16 \mu \mathrm{g} / \mathrm{ml}$ in serum-free RPMI-1640 medium (Gibco). Lipofectamine ${ }^{\mathrm{TM}} 2000$ (Invitrogen) was diluted in serum-free RPMI-1640 medium $(40 \mu \mathrm{l} / \mathrm{ml})$ and then mixed with an identical volume of plasmid solution, resulting in lipid-DNA complexes.

HeLa and L929 cells (ATCC) were cultured in RPMI-1640 medium supplemented with $10 \%$ fetal bovine serum (FBS; Hyclone), $100 \mathrm{IU} / \mathrm{ml}$ penicillin, and $100 \mu \mathrm{g} / \mathrm{ml}$

Table 2: Primers used for cloning psrpk ${ }^{d}$ and psrpk $^{m}$

\begin{tabular}{lll}
\hline & DNA fragmants & Sense and anti-sense primers \\
\hline psrpk $^{d}$ & 1 95I & F4/5'-GCAAAAAAGGAGATCTCCGGTGGCC-3' \\
& $969 \sim 1278$ & 5 '-GGAGATCTCCTTTTTGCTATGATAAGACAGAT-3'/RI \\
psrpk $^{m}$ & $959 \mathrm{~A} \rightarrow 959 \mathrm{C}$ & F4/5'-GCAAAAAAGGAGATCTCCGGTGGCC-3' \\
& & 5'-GGAGATCTCCTTTTTTGCCCAAAACAGGAG-3'/R3
\end{tabular}


streptomycin at $37^{\circ} \mathrm{C}$ in a $5 \% \mathrm{CO}_{2}$ incubator. The cells were harvested while they were in the logarithmic phase and $1.5 \mathrm{ml}$ culture (approximately $1 \times 10^{5}$ cells) was seeded into a $35-\mathrm{mm}$ plate with a coverslip and cultured for $24 \mathrm{~h}$. The medium was replaced with serum-free RPMI1640 medium, and the cells were cultured for $1 \mathrm{~h}$ to initiate transfection. The cells were overlaid with $200 \mu$ lipidDNA complexes and cultured for $5 \mathrm{~h}$. The medium was replaced with $1.5 \mathrm{ml}$ of $10 \%$ FBS medium, and the cells were further cultured for $48 \mathrm{~h}$.

Staining of cells with 4',6-diamidino-2-phenylindole (DAPI) The HeLa cells were transfected with pECFP-psrpk, and L929 cells were transfected with pECFP-psrpk, pDsRedpsrpk, pDsRed-tp5 pDsRed-tp5, pDsRed-psrpk and pDsRed-psrpk $k^{m}$ as described above, respectively. The transfected cells were incubated on a coverslip for $48 \mathrm{~h}$, following which DAPI staining (Bitian) was performed for 5 min. The cells were washed 3 times (each wash, $5 \mathrm{~min}$ ) with D-Hands solution to remove DAPI. Fluorescence was visualized under a fluorescence microscope (Olympus
BX51, 400x) or a confocal microscope (Leica TCS SP2, $1000 \times$ ) at $465 \sim 495 \mathrm{~nm}$ (green) or 560 600 nm (red) in order to observe the distribution of the fluorescent fusion protein in the cells.

\section{Three-Dimensional Structure Modeling of PSRPK}

The three-dimensional (3-D) PSRPK structure was modeled by the Swiss Institute of Bioinformatics program SWISS-MODEL, available at http://swiss model.expasy.org/, and the results analyzed by the DeepView program [17].

\section{Results}

PSRPK was mainly localized in the nucleus of mammalian cells

The DAPI-stained nucleus of cells HeLa and L929 transfected with pECFP-psrpk were clearly observed under UV excitation (Figure 2a, b). Confocal microscopy showed that the distribution of cyan fluorescent protein (CFP)PSRPK was non-uniform and that this protein was

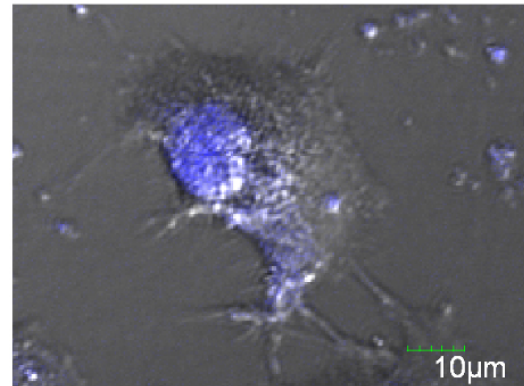

a: Nucleus of a HeLa cell stained with DAPI

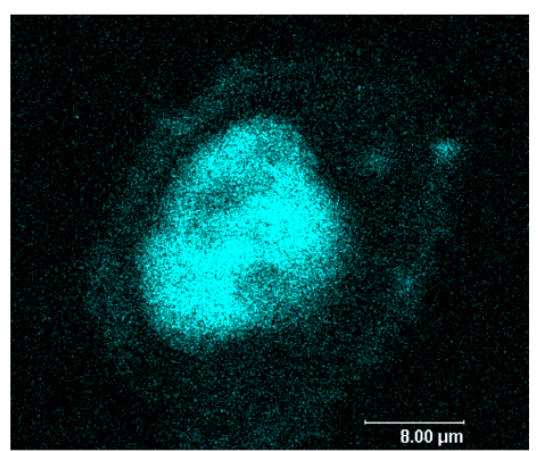

c: distribustion of CFP-PSRPK in a HeLa cell

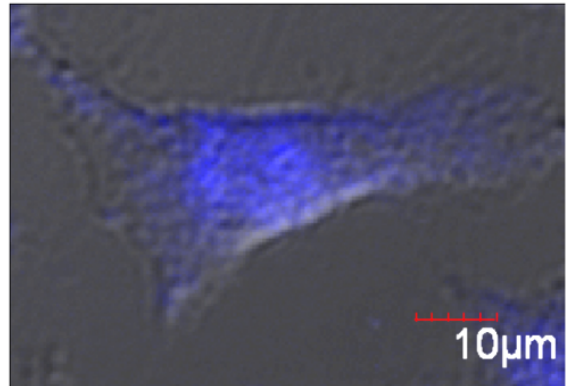

b: Nucleus of a L929 cell stained with DAPI

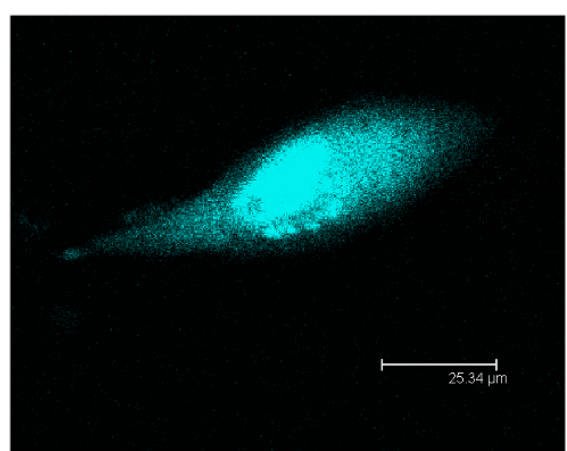

$\mathrm{d}$ : distribustion of CFP-PSRPK

in a L929 cell

\section{Figure 2}

Schematic diagrams of truncated PSRPKs compared with PSRPK. TP I TP7: Peptides containing the PSRPK aa 29 426, I 195, 196 426, 259 426, 197 317, 197 343, and 329 426, respectively; TP8: peptides containing 197 3 I7 aa combined with 329 426 aa; TP9: peptides containing I 3I7 aa combined with 329 426 aa. N: acidic divergent motif; I 28 aa; CD I: conserved domain I; 29 195 aa; CD2: conserved domain 2; 259 426 aa; spacer sequence: divergent motif between the 2 conserved domains; 196 258 aa. NLS: ${ }^{318}$ PKKGDKYDKTD ${ }^{328}$. 

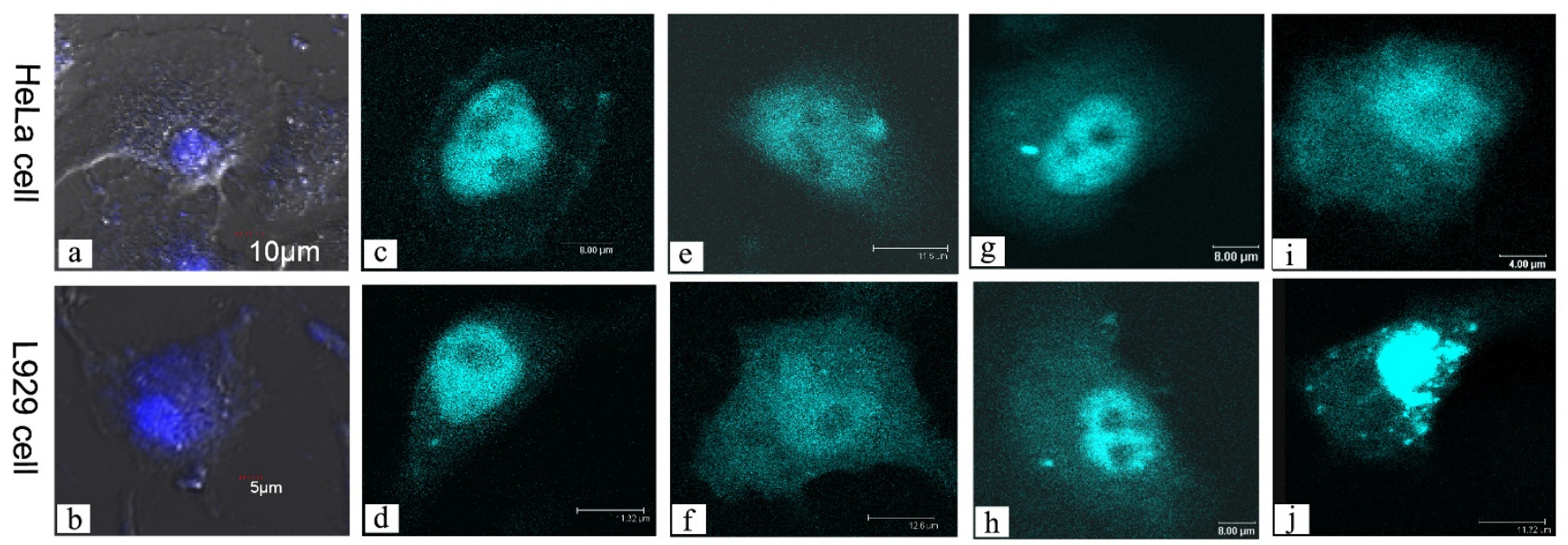

DAPI-stained

CFP-TP1 expression

CFP-TP2 expression

CFP-TP3 expression CFP-TP4 expression

Figure 3

Distribution of CFP-TPs in the mammalian cells HeLa and L929 observed under a confocal microscope (TCS SP2, Leica). The images a and b show DAPI staining nucleus of cells HeLa and L929; the images $c$ and $d, e$ and $f, g$ and $h$, and $i$ and $j$ show CFP-TPI, CFP-TP2, CFP-TP3, and CFP-TP4 expressions in the HeLa and L929 cells, respectively.

expressed mainly in the nucleus of the HeLa (Figure 2c) and L929 (Figure 2d) cells.

\section{Existence of an NLS on the C-terminal conserved domain of PSRPK}

The expression and distribution of CFP fusion TP1, TP2, TP3, and TP4 in mammalian cells were observed by con- focal microscopy. Compare with the DAPI staining of the nucleus (Figure 3a, b), the distribution of CFP-TP1 in HeLa (Figure 3c) and L929 (Figure 3d) cells was similar to that of CFP-PSRPK, i.e., both mainly accumulated in the nucleus. This suggested that the deletion of $\geq \mathrm{N}$ had no significant effect on the nuclear accumulation of PSRPK. The distributions of CFP-TP3 and CFP-TP4 in HeLa (Fig-
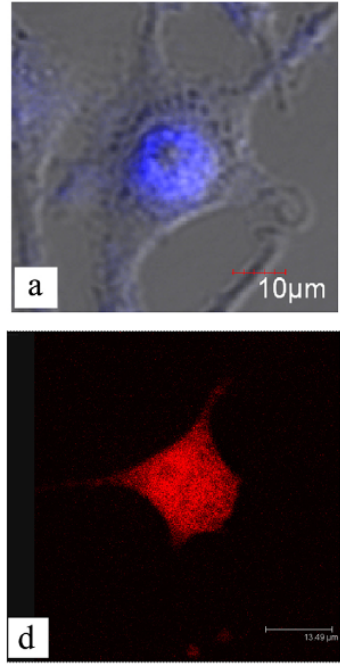
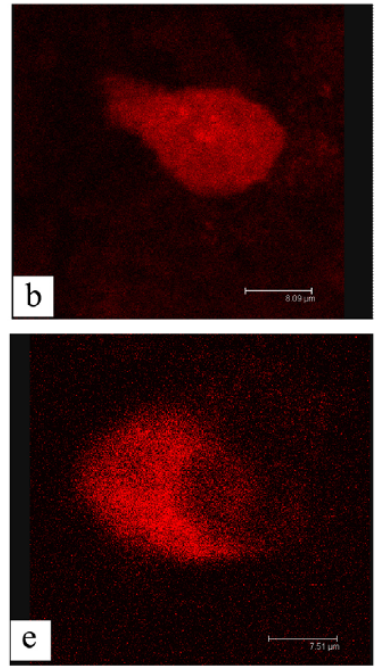
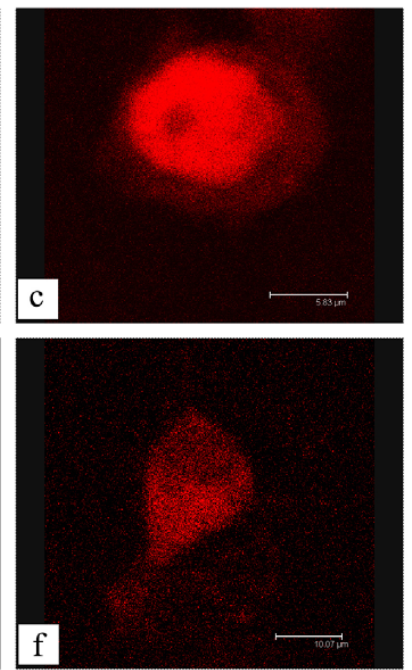

\section{Figure 4}

Distribution of RFP-TP5 RFP-TP9 in L929 cells observed by a confocal microscope (TCS SP2, Leica). Only RFPTP6, which contained ${ }^{318 P K K G D K Y D K T D ~}{ }^{228}$, accumulated in the nucleus. a: Nucleus of a pDsRed-tp6-transfected L929 cells stained with DAPI and observed under a BX5I fluorescent microscope (Olympus, 400x), while TP8 and TP9 are peptides obtained by the deletion of ${ }^{318}$ PKKGDKYDKTD 328 from TP3 and PSRPK, respectively. b, c, d, e, and f: Expressions of RFPTP5 RFP-TP9 in L929 cells. 
ure 3g, i) and L929 (Figure 3h, j) cells were similar to those of CFP-PRPK and CFP-TP1, i.e., both mainly accumulated in the nucleus. This indicated that PSRPK continued to accumulate in the nucleus despite the deletion of $\geq$ $\mathrm{N}, \mathrm{CD} 1$, and the spacer sequence and that CD2 contains an NLS peptide. Unlike the distributions of CFP-PSRPK, CFP-TP1, CFP-TP3, and CFP-TP4, CFP-TP2 in HeLa (Figure $3 e$ ) and L929 (Figure 3f) cells exhibited diffuse distribution. The results above indicated that the $\geq \mathrm{N}, \mathrm{CD} 1$, and spacer sequence deletions did not influence nuclear localization of PSRPK and that an NLS was located in the C-terminal conserved domain of PSRPK.

\section{PSRPK NLS was located in the ${ }^{318}$ PKKGDKYDKTD 328 sequence}

Confocal microscopy revealed that RFP-TP5 and RFP-TP7 were uniformly distributed in the L929 cells (Figure 4b, d), indicating that there was no NLS in the TP5 and TP7 sequences. However, RFP-TP6 mainly accumulated in the nucleus (Figure 4c), indicating that an NLS existed in TP6. By comparing the amino acid sequences of TP5, TP6, and TP7, we primarily confirmed that PSRPK NLS was located in 318PKKGDKYDKTD ${ }^{328}$. RFP-TP8 and RFP-TP9 were mainly distributed in the cytoplasm (Figure $4 \mathrm{e}, \mathrm{f}$ ); this further indicated that PSRPK and TP3 lost the ability of nuclear localization after the deletion of the abovementioned sequence. Thus, we could confirm that 318PKKGDKYDKTD ${ }^{328}$ was the NLS sequence of PSRPK.

\section{The NLS sequence of PSRPK contained a $\Omega$-loop motif}

Analysis of the secondary structure of 318PKKGDKYDKTD 328 within PSRPK by DNASIS v2.5 Demo revealed that it was a $\beta$-turn (Figure 5A). Based on the crystal structure of SRPK1 and Sky1p [17], the tertiary structure of PSRPK (Figure 5B) was predicted using SWISS-MODEL software [18-20]. Figure 5B shows that the

284 PEVI LGQKWDT T IDMWS LACMVF E LATGDLLFCPKKGDKYDKTDDHLALMI E LLGRMPRSF 345 SHHHHTT T T HHHHTT T T HHHH HHHHTTTTTSSTTT T TTT TTT TTT TTHHHHHHHHHTTTTT

A. Secondary structure of PSRPK NLS and its contiguous sequences

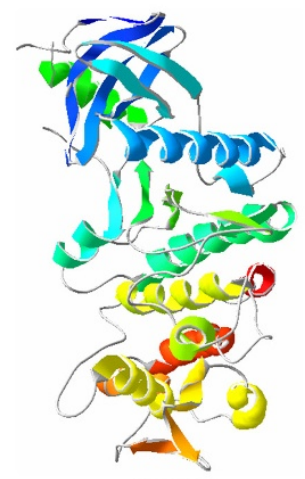

SRPK1

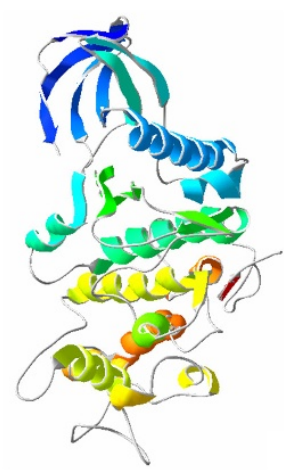

Skylp

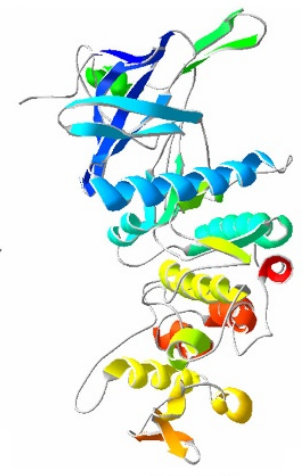

PSRPK

B. Overall structure of SRPK1 and Sky1p and the overall structure of PSRPK that mimics the structures of SRPK1 and Skylp

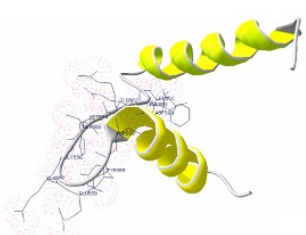

NLS of PSRPK

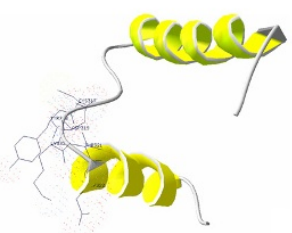

NLS ${ }^{\mathrm{d}}$ of PSRPK

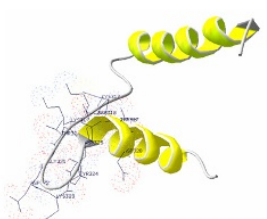

$\mathrm{NLS}^{\mathrm{m}}$ of PSRPK

C. Partial mimic structures of NLSs of PSRPK, PSRPK ${ }^{\mathrm{d}}$, and PSRPK ${ }^{\mathrm{m}}$

\section{Figure 5}

A: Secondary structure of PSRPK NLS, as predicted by DNASIS v2.5 Demo. The capital H, S and T blow the sequence represent the corresponding amino acid above has more probability to form helix, sheet or turn. B: The overall structure of PSRPK that mimics the structures of SRPKI and Skylp, as predicted by SWISS-MODEL online and displayed by SWISS-Pdb viewer compared with the overall structure of SRPKI and Skylp [17-20]. C: NLS, the NLS of PSRPK; NLSd, the NLS of PSRPK without ${ }^{318 P K K G D K}{ }^{323}$; NLS ${ }^{m}$, the NLS of PSRPK with the $\mathrm{K}^{320} \rightarrow \mathrm{T}^{320}$ mutation. 
structure ${ }^{318}$ PKKGDKYDKTD 328 mimics that of the corresponding sequences of SRPK1 and Sky1p. All the 3 sequences are located in the C-terminal loop motif. The spatially near $\mathrm{K}^{320}$ and $\mathrm{D}^{325}$ may form a salt-bridge via their side chains, causing the 318 PKKGDKYDKTD ${ }^{328}$ to form a stable $\Omega$-loop. This structure of 318PKKGDKYDKTD ${ }^{328}$ changes after ${ }^{318}$ PKKGDK $^{323}$ deletion or $\mathrm{K}^{320} \rightarrow \mathrm{T}^{320}$ mutation, as predicted by SWISSMODEL software (Figure 5C). ${ }^{318}$ PKKGDK $^{323}$ deletion causes damage to the $\Omega$-loop motif, and $\mathrm{K}^{320} \rightarrow \mathrm{T}^{320}$ mutation damages the salt-bridge in the $\Omega$-loop.

The fluorescent fusion proteins PSRPK $^{\mathrm{d}}$ and $\mathrm{PSRPK}^{\mathrm{m}}$ in the L929 cells are shown in Figure 6. Compare with the DAPI staining of the nuclear (Figure 6B1a, B2a), the fluorescent signal of RFP-PSRPK ${ }^{d}$ and RFP-PSRPKm was observed in cytoplasm (Figure 6B1b, B2b). Further, confocal microcopy revealed that RFP-PSRPK ${ }^{d}$ and RFPPSRPK $^{\mathrm{m}}$ were mainly distributed in the cytoplasm (Figure 6B1c, B2c); this indicated that the 318 PKKGDK $^{323}$ deletion or $\mathrm{K}^{320} \rightarrow \mathrm{T}^{320}$ mutation destroyed the structure of PSRPK NLS, resulting in PSRPK losing its ability of nuclear localization.

\section{Discussion \\ PSRPK has an NLS sequence}

Ding et al. [11] studied the cell localization of mammalian SRPKs and found that almost all SRPKs without spacer sequences are accumulated in the nucleus, indicating that there is a cytoplasm localization signal in the spacer sequence between the conserved domains. Kuroyanagi et al. [5] speculated that mouse SRPK1 might have 2 potential NLSs that are located in 11 21 aa and 265 277 aa; thus, mSRPK2 may have a potential NLS in 264 276 aa. However, direct evidence of NLS sequences in SRPK family members has been lacking thus far. Laser scanning confocal microscopy revealed that RFPs of default PSRPKs containing 318PKKGDKYDKTD ${ }^{328}$ mainly accumulated in the nucleus of mammalian cells, while the PSRPKs in which the abovementioned sequence was deleted did not accumulate in the nucleus. This indicated that the NLS of PSRPK was located in the ${ }^{318}$ PKKGDKYDKTD ${ }^{328}$ sequence of the C-terminal conserved domain.

Further, Ding et al. [11] showed that mammalian SRPKs were mainly distributed in the cytoplasm. However, the

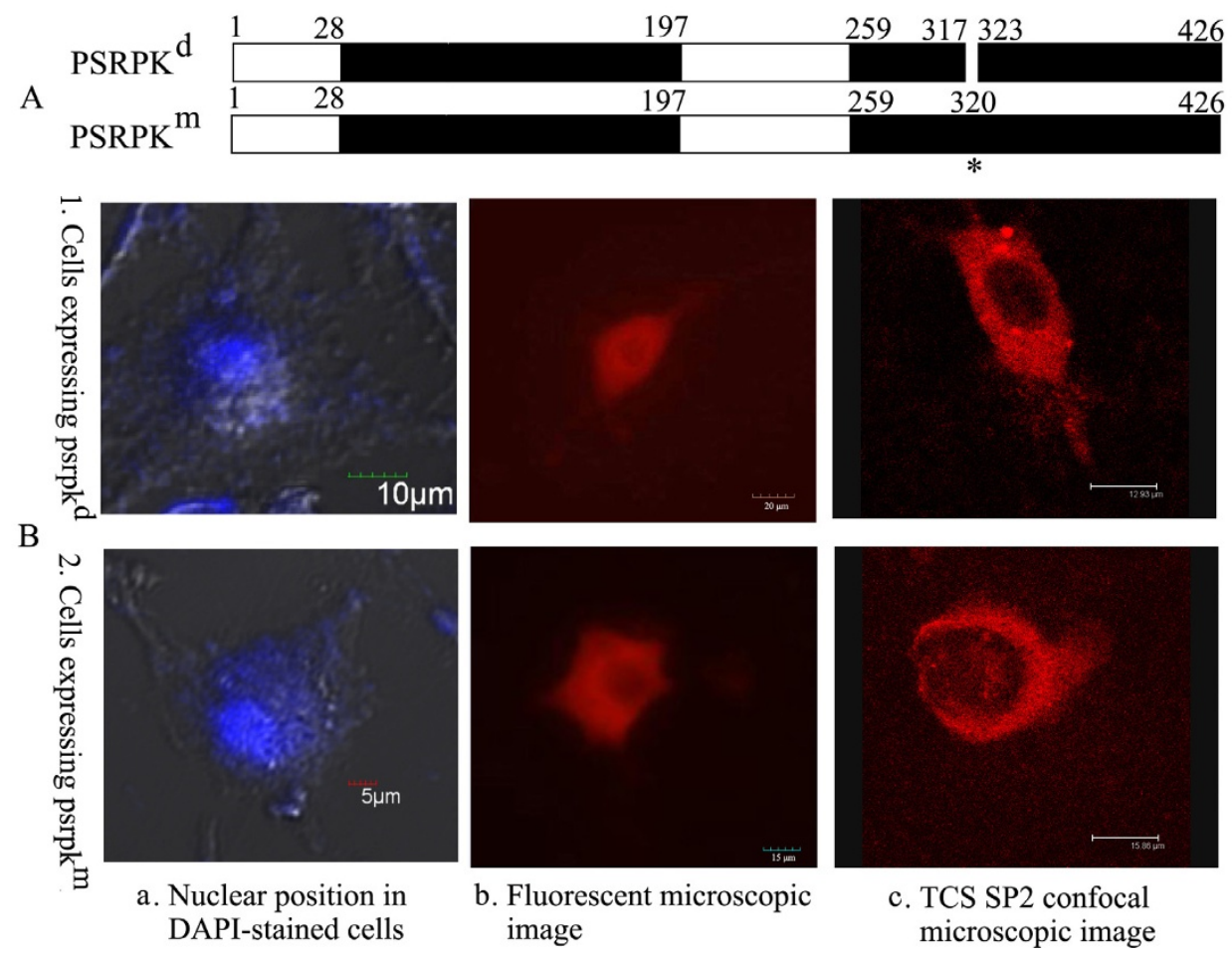

\section{Figure 6}

A: Schematic diagrams of default PSRPK and mutant PSRPK compared with PSRPK. B: Distribution of RFPPSRPK $^{d}$ and RFP-PSRPK ${ }^{m}$ expression in L929 cells. PSRPKd, default PSRPK without ${ }^{318 P K K G D K ~}{ }^{323}$; PSRPK ${ }^{m}$, mutant PSRPK with the $\mathrm{K}^{320} \rightarrow \mathrm{T}^{320}$ mutation. I and 2: Distribution of RFP-PSRPKd and RFP-PSRPKm expressions in L929 cells observed under a confocal microscope (TCS SP2, Leica) compared with their position in the nucleus in DAPI-stained cells observed under a BX5I fluorescent microscope (Olympus, 400x). 
results obtained in our study showed that PSRPK was mainly expressed in the nucleus of the mammalian cells. A comparison of the primary structure of PSRPK and other SRPKs revealed that the conserved domains were almost identical; however, the nonconserved $\geq N$ and spacer sequence differed among the kinases. A cytoplasm localization signal was present in the spacer sequences of mammalian SRPKs. Further, the spacer sequence of PSRPK was considerably smaller than that of mammalian SRPKs. This difference might be the major reason why PSRPK cannot anchor itself in the mammalian cytoplasm.

\section{Function of the PSRPK NLS is related to the loop motif}

A classic NLS (cNLS) comprises a monopartite or bipartite signal. A monopartite NLS contains 1 cluster of basic residues, while a bipartite NLS contains 2 clusters of basic residues [21]. Similar to the sequence feature of a monopartite NLS, the NLS of PSRPK is rich in basic residues. A monopartite NLS sequence that is located at the terminal of a protein is usually in a coil, while that located in the interior of a protein is usually in a loop. The PSRPK NLS is also located in the basic loop between $2 \alpha$-helixes (Figure 5). The close side chains of $\mathrm{K}^{320}$ and $\mathrm{D}^{325}$ form a salt bridge, thus forming a stable NLS $\Omega$-loop motif (Figure 5C). The experimental results showed that PSRPK lost its nuclear localization ability following the deletion of 318 $\mathrm{PKKGDK}^{323}$ or mutation from $\mathrm{K}^{320}$ to $\mathrm{T}^{320}$. The deletion or mutation destroyed the $\Omega$-loop motif of PSRPK, thus suggesting that the loop structure of PSRPK NLS controls the nuclear localization of PSRPK. The ${ }^{318}$ PKKGDKYDKTD 328 sequence, which corresponds to the nonconserved sequences of SRPK1 and Sky1p, is located in the loop of an HLHM $[18,19]$ (Figure 5B). Therefore, studies on NLSs of SRPKs should focus on the loops in HLHMs.

\section{Conclusion}

In this study, by truncation of PSRPK protein, deletion of and single amino acid substitution in a putative NLS and transfection of mammalian cells, we demonstrated that the ${ }^{318}$ PKKGDKYDKTD ${ }^{328}$ peptides localized in the C-terminal conserved domain of PSRPK with the $\Omega$-loop structure could play a crucial role in the NLS function of PSRPK.

\section{Authors' contributions}

SL carried out the design of the study, participated in all studies, and drafted the manuscript. ZZ carried out the most of the tests. ZL carried out confocal microscopy scanning. QO constructed partial plasmids. JZ participated all of the tests. ST participated in the study, and proofreading the manuscript. MX, conceived of the study, participated in its design and coordination.

\section{Acknowledgements}

The work was supported by the National Natural Science Foundation of China (30470 I I3), Natural Science Foundation of Guangdong Province (040II314)

\section{References}

I. Aubol BE, Chakrabarti S, Ngo J, Shaffer J, Nolen B, Fu XD, Ghosh G, Adams JA: Processive phosphorylation of alternative splicing factor/splicing factor 2. Proc Natl Acad Sci USA 2003, 100: I 260I-I2606.

2. Gui JF, Lane WS, Fu XD: A serine kinase regulates intracellular localization of splicing factors in the cell cycle. Nature 1994, 369:678-682.

3. Gui JF, Tronchere H, Chandler SD, Fu XD: Purification and characterization of a kinase specific for the serine- and argininerich pre-mRNA splicing factors. Proc Natl Acad Sci USA 1994, 91:10824-10828.

4. Wang HY, Lin W, Dyck JA, Yeakley JM, Songyang Z, Cantley LC, Fu XD: SRPK2: a differentially expressed SR protein-specific kinase involved in mediating the interaction and localization of pre-mRNA splicing factors in mammalian cells. J Cell Biol 1998, 140:737-750.

5. Kuroyanagi N, Onogi H, Wakabayashi T, Hagiwara M: Novel SRprotein-specific kinase, SRPK2, disassembles nuclear speckles. Biochem Biophys Res Commun 1998, 242:357-364.

6. Tang Z, Yanagida M, Lin RJ: Fission yeast mitotic regulator Dsk I is an SR protein-specific kinase. J Biol Chem 1998, 273:5963-5969.

7. Siebel CW, Feng L, Guthrie C, Fu XD: Conservation in budding yeast of a kinase specific for SR splicing factors. Proc Natl Acad Sci USA 1999, 96:5440-5445.

8. Kuroyanagi $\mathrm{H}$, Kimura T, Wada K, Hisamoto N, Matsumoto K, Hagiwara M: SPK-I, a C. elegans SR protein kinase homologue, is essential for embryogenesis and required for germline development. Mech Dev 2000, 99:5 I-64.

9. Portal D, Lobo GS, Kadener S, Prasad J, Espinosa JM, Pereira CA, Tang Z, Lin RJ, Manley JL, Kornblihtt AR, Flawiá MM, Torres HN: Trypanosoma cruzi TcSRPK, the first protozoan member of the SRPK family, is biochemically and functionally conserved with metazoan SR protein- specific kinases. Mol Biochem Parasitol 2003, I 27:9-2I.

10. de la Fuente van Bentem S, Anrather D, Roitinger E, Djamei A, Hufnagl T, Barta A, Csaszar E, Dohnal I, Lecourieux D, Hirt H: Phosphoproteomics reveals extensive in vivo phosphorylation of Arabidopsis proteins involved in RNA metabolism. Nucleic Acids Res 2006, 34:3267-3278.

II. Ding JH, Zhong XY, Hagopian JC, Cruz MM, Ghosh G, Feramisco J, Adams JA, Fu XD: Regulated cellular partitioning of SR protein-specific kinases in mammalian cells. Mol Biol Cell 2006, I7:876-885.

12. Koizumi J, Okamoto Y, Onogi H, Mayeda A, Krainer AR, Hagiwara M: The subcellular localization of SF2/ASF is regulated by direct interaction with SR protein kinases (SRPKs). J Biol Chem 1999 , 274: I||25-I||3|.

13. Misteli T, Cáceres JF, Clement JQ, Krainer AR, Wilkinson MF, Spector $D L$ : Serine phosphorylation of SR proteins is required for their recruitment to sites of transcription in vivo. J Cell Biol 1998, 143:297-307.

14. Yeakley JM, Tronchère H, Olesen J, Dyck JA, Wang HY, Fu XD: Phosphorylation regulates in vivo interaction and molecular targeting of serine/arginine-rich pre-mRNA splicing factors. J Cell Biol 1999, I45:447-455.

15. Tang Z, Tsurumi A, Alaei S, Wilson C, Chiu C, Oya J, Ngo B: Dsk Ip kinase phosphorylates SR proteins and regulates their cellular localization in fission yeast. Biochem J 2007, 405:2 I-30.

16. Liu SD, Kang K, Zhang JH, Ouyang QL, Zhou ZL, Tian SL, Xing M: A novel SR protein kinase from Physarum polycephalum phosphorylates specificlly on RS domain of human SR protein ASF/SF2. Acta Biochimica et Biophysica Sinica 2009, 4 I(8):657-667.

17. Schwede T, Kopp J, Guex N, Peitsch MC: SWISS-MODEL: an automated protein homology- modeling server. Nucleic Acids Research 2003, 31:338I-3385.

18. Lukasiewicz R, Velazquez-Dones A, Huynh N, Hagopian J, Fu XD, Adams J, Ghosh G: Structurally unique yeast and mammalian serine-arginine protein kinases catalyze evolutionarily con- 
served phosphorylation reactions. J Biol Chem 2007, 282:23036-23043.

19. Nolen B, Yun CY, Wong CF, McCammon JA, Fu XD, Ghosh G: The structure of Sky lp reveals a novel mechanism for constitutive activity. Nat Struct Biol 200I, 8:176-183.

20. Ngo JC, Gullingsrud J, Giang K, Yeh MJ, Fu XD, Adams JA, McCammon JA, Ghosh G: SR protein kinase I is resilient to inactivation. Structure 2007, I5:123-133.

21. Lange A, Mills RE, Lange CJ, Stewart M, Devine SE, Corbett AH: Classical nuclear localization signals: definition, function, and interaction with importin alpha. J Biol Chem 2007, 282:510I-5I05.

Publish with Bio Med Central and every scientist can read your work free of charge

"BioMed Central will be the most significant development for disseminating the results of biomedical research in our lifetime. "

Sir Paul Nurse, Cancer Research UK

Your research papers will be:

- available free of charge to the entire biomedical community

- peer reviewed and published immediately upon acceptance

- cited in PubMed and archived on PubMed Central

- yours - you keep the copyright

Submit your manuscript here:

http://www.biomedcentral.com/info/publishing_adv.asp 